\title{
Feasibility study of innovative regional turboprop: an overview of the European project IRON
}

\author{
Salvatore Corcione ${ }^{1, *}$, Vittorio Trifari ${ }^{1}$, Fabrizio Nicolosi $^{1}$, Vincenzo Cusati ${ }^{1}$, Danilo \\ Ciliberti $^{1}$ and Pierluigi Della Vecchia ${ }^{1}$ \\ ${ }^{1}$ University of Naples Federico II, Industrial Engineering Department, Via Claudio 21, Naples, Italy
}

\begin{abstract}
This paper deals with the research activities performed by the Design of Aircraft and Flight technologies group from the University of Naples Federico II within the European funded project IRON (Innovative turbopROp configuratioN). The research project is addressed to the feasibility study of an innovative high-capacity turboprop which is supposed to be competitive with respect to regional jets on short/medium range. This paper wants to provide some design considerations that must be addressed to design a high capacity turboprop, and to illustrate the configuration assessment through a Multi-Disciplinary Analysis and Optimization process performed to design such an innovative platform. A three-lifting surface configuration has been identified as the most promising layout for such a regional aircraft. Moreover, this paper wants to focus on some criticalities and design challenges that have been faced into designing a three-lifting platform.
\end{abstract}

\section{Introduction}

An in-depth market analysis of the regional aircraft segment has revealed that nowadays the increase in oil price, the huge growth of air transport traffic, and the increasing attention to the aircraft environmental footprint has led to considerable interest of specialists in new configurations of regional transport aircraft. Furthermore, the major airlines in this segment have been demanding a replacement for several hundred heritage airplanes, currently in service around the world, in the 20-150 seats categories, as many are now coming to the end of their useful commercial life. The climate change and the increasing lack of resources claim for a clear reduction of the aviation impact on citizens and the environment. The integration of innovative and affordable technologies in future aircraft platforms plays a key role to increase the appeal and the benefits for both customers and airlines. Regional aircraft are playing an increasing role in the evolution of the airline operations. For many years, this growth has been faced by a wide adoption of regional jets (RJ). Their success can be largely attributed to their popularity with travellers, who prefer them because they are more comfortable and faster than Turboprops (TPs). Despite the RJ success, TP engines

*Corresponding author: salvatore.corcione@unina.it 
are 10 up to $30 \%$ more efficient than jet engines in cruise conditions. TPs typically derive $85 \%$ of their thrust from a propeller, while jet exhaust provides the remaining thrust. Their Specific Fuel Consumption (SFC) is the result of the propellers capability to accelerate large amounts of air at low speeds. The efficiency of a propeller decreases with increasing airspeed and altitude limiting the operation of TPs to Mach numbers below 0.7, also considering compressibility effects at blades tip, and altitudes below $7.6 \mathrm{~km}$.

Is it possible to fill the gap between RJs and TPs by adopting new technologies or innovative aircraft configurations?

Nowadays several research works are focused on the investigation of new regional platforms which cope with ever more stringent performance, costs and emissions requirements. Innovation, in the regional TPs field, can come from improved power plants (e.g. open rotors, low noise propeller designs, etc.), innovative materials and from unconventional aircraft concepts (e.g. Box-Wing, Strut-Braced Wings, Blended Wing Body, Three-Lifting Surfaces, etc.).

The research activities performed within the IRON project are properly linked to the above question.

\subsection{The IRON project}

The IRON project has been funded in the frame of the second call for partners of Clean Sky 2 IADP (Innovation Area Development Partnership) Regional topic. The Italian Aerospace Research Center $\left(\mathrm{CIRA}^{\dagger}\right)$ is the coordinator of the project, Leonardo Company* is the Topic Leader and 10 core partners from several European countries are involved. The research program is addressed to the performance improvement of regional aircraft within the 90-150 passenger segment. In this research project, two different turboprop aircraft configurations will be studied: a conventional aircraft configuration capable of carrying up to 90 passengers and a non-conventional aircraft configuration with a capacity of 130 passengers.

This article deals with the research activities performed by $\mathrm{DAF}^{\S}$ research group into the feasibility study of the unconventional turboprop configuration.

\subsection{Innovative high-capacity turboprop configuration}

In designing a high-capacity turboprop aircraft several aspects must be considered, dealing with the aircraft layout. Generally, large turbo-propeller aircraft (with about 70 seats) have a maximum take-off weight of about 23-28 tons with an empty weight slightly higher than $50 \%$ of the maximum take-off weight (about 13-17 tons). Turboprops have a straight tapered high-wing configuration with a surface of about $60-70 \mathrm{~m}^{2}$ and a span of approximately 27-30 meters, which means an aspect ratio in the range 11-12. A T-tail configuration is adopted, with a ratio between tails and wing surfaces close to 0.2 . Also, the fuselage length and fuselage fineness ratio are comparable for each aircraft, between 10 and 12. The high-wing configuration with under-wing engines installation represents the state of the art for existing large turboprop. The main reason behind this layout is to have an easy cabin access and a better aircraft clearance due to large propeller diameter. Furthermore, the under-wing engines installation provides a lighter wing structure (engine mass loading relief) and a contained Centre of Gravity (CG) excursion which leads to a lower horizontal tail download to trim the aircraft at the most forward centre of gravity position. However,

\footnotetext{
${ }^{\dagger}$ www.cira.it [Retrieved August 2019]

*www.leonardocompany.com [Retrieved August 2019]

$\S$ Website: www.daf.unina.it [Retrieved August 2019]
} 
such a configuration could be not suitable for future high capacity turboprop configuration with increased passenger capacity (130-150 seats). Increasing the number of passengers means to increase the aircraft weight as well and this leads to a larger wing area to keep similar ground performance. To avoid a large decay of the aerodynamic efficiency, the wing aspect ratio should be kept in the range of 11-12 which means to increase the wingspan affecting, this way, the landing gears size and its position. A larger wheel track is required to ensure ground stability while an increased maximum take-off weight $\left(\mathrm{W}_{\mathrm{TO}}\right)$ demands for a heavier landing gear structure. For high-wing configuration, two landing gears installations are possible: nacelle-mounted or fuselage-mounted with pods.

These two solutions may provide several issues if used for high capacity TPs. The nacelle-mounted landing gear will require for a very long and heavy leg which may be difficult to be retracted inside the nacelle, while the fuselage mounted landing gear could require very large and heavy pods to ensure the required wheel track. Moreover, for both these layouts the length of landing gear legs must be increased to achieve a sufficient value of the upsweep angle due to a longer fuselage. Unless new technological improvements concerning the landing gears design, the abovementioned solutions could be unfeasible for high-capacity TPs. A low-wing configuration could solve this issue. However, the need for a very large propeller diameter (about 12-14 ft) makes impossible to reach the required engine clearance from the ground. Thus, a low wing with under-wing engine installation is unfeasible. For this reason, new TP configurations should be characterized by rear mounted engine installation. This can lead to a more efficient wing, thanks to a large laminar flow extension, together with a lower cabin noise level (engines far from the cabin). In addition, the wing without engine nacelle and propeller interferences will be characterized by a more efficient high-lift system (flap) with a possible increase in aircraft maximum lift coefficient, which will positively affect ground performance. Conversely, a rear engine installation will lead to a wing weight increment (no engine mass relief on the wing loading).

\subsection{Top-Level Aircraft Requirements (TLARs) and baseline configuration}

Top Level Aircraft Requirements (TLAR) have been issued by Leonardo company, which also provided the aircraft maximum take-off weight and wing area. TLAR are very challenging: a cruise Mach number of 0.64 (or higher) at 30000 feet, with a moderately high maximum lift coefficient and low drag to achieve a cruise aerodynamic efficiency about 18. A maximum lift coefficient of 2.4 and 3.0 in take-off and landing conditions are respectively required. Moreover, it is expected that the inner wing airfoil have $18 \%$ relative thickness to reduce the wing weight and to allow the storage of the landing gear. In Fig. 1, the reference aircraft is illustrated. The baseline shows a wing area of about $105 \mathrm{~m}^{2}$ with an aspect ratio equal to 12 , the fuselage length is about $38 \mathrm{~m}$.

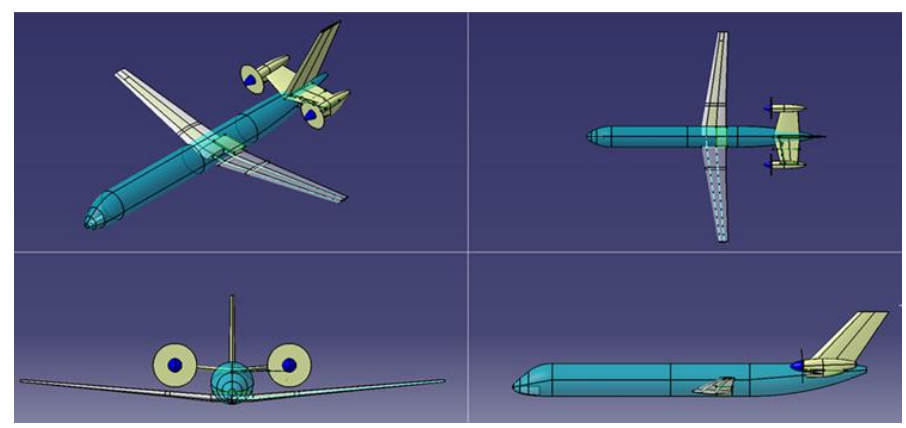

Fig. 1. Baseline aircraft configuration. 


\section{Configuration assessment}

The rear propeller installation must be carefully investigated to have a reliable prediction of aircraft tail aerodynamics, considering also for the engine installation and the influence of the propeller on the horizontal tail sizing. This configuration can lead to a very large Center of Gravity (CG) excursion which can also affect aircraft performance. A wide CG excursion could imply a very big horizontal tail to trim the aircraft in the most rearward CG positions resulting in a reduction of the maximum lift capabilities; while in the most forward CG position the longitudinal static margin could be very high implying a very large download on the tail to trim the aircraft. This latter will reduce the cruise efficiency affecting the fuel burned and aircraft Direct Operating Costs (DOC). One possible solution could be a reasonable reduction of the CG excursion (limitation of aircraft operability with low passenger number) complying with typical aircraft missions, as outlined by authors in [1]. A possible solution to overcome this drawback could be a three lifting surfaces configuration. In this case the third lifting surface (the canard) will extend the design space allowing to cope with both cruise efficiency and maximum lift capabilities of the aircraft. In fact, the third surface will augment the whole aircraft maximum lift coefficient, while in cruise conditions the canard surface will help in reducing the horizontal tail download required to trim the aircraft leading to a higher cruise efficiency if compared to the conventional configuration [1].

\subsection{Multi-Disciplinary Analysis and Optimization (MDAO)}

The main tool used to fulfill the MDAO task has been JPAD software developed at Industrial Engineering Department of the University of Naples Federico II by the DAF group [1][2]. JPAD is also provided with all the current state of-the-art metaheuristic optimization algorithms thanks to the use of a dedicated external library named MOEA Framework, a free and open source Java library for Multi Objective Evolutionary Algorithms (MOEAs). Using both these algorithms, the JPAD optimization module can easily solve complex MDAO.

Starting from TLARs described in section 1.3, three possible aircraft configurations, have been investigated (see Fig. 2): configuration 1 for which no limitations on the operating range of CG excursion have been imposed; configuration 2 for which a large limitation to the operating range has been fixed by limiting the maximum rearward CG position and configuration 3 in which the third lifting surface has been added and a very limited restriction to the operating CG range has been assigned. A sensitivity analysis, for each configuration, has been performed by varying the main design parameters of lifting surfaces (span, area, aspect ratio, longitudinal position, etc.). The cloud of solution points has been used to build up a response surface (one per configuration) used to perform the optimization task. At the end of the process, charts of all possible combinations of Pareto fronts as well as the complete set of the optimal design variables and objectives have been produced. According to the chosen number of design parameters, more than 7000 different combinations (each combination represents an aircraft) have been generated and analyzed to define the response surface on which perform the optimization process, see Fig. 3. The Pareto front, in this optimization problem is a multidimensional frontier, according to the chosen design parameters. Targets of the optimization process have been the cruise parameter $\mathrm{W} / \mathrm{E}$, the take-off and landing factors $\mathrm{W} /\left(\mathrm{S}_{\mathrm{w}}{ }^{*} \mathrm{C}_{\mathrm{Lmax}}\right)$. To ensure the aircraft stability, a static margin of $5 \%$ has been assigned as constraint. Fig. 2 shows the comparison between the three optimized configurations and their relative load and balance diagram, while Table 1 provides a summary of the main results. To ensure the required stability, Configuration 1 is characterized by a very large horizontal tail area $\left(\mathrm{S}_{\mathrm{H}}=56 \mathrm{~m}^{2}\right)$ 
about $51 \%$ of the wing area. This provides an increment of both the maximum take-off weight and induced drag which lead to the lowest cruise efficiency among the three solutions, see Table 1 .
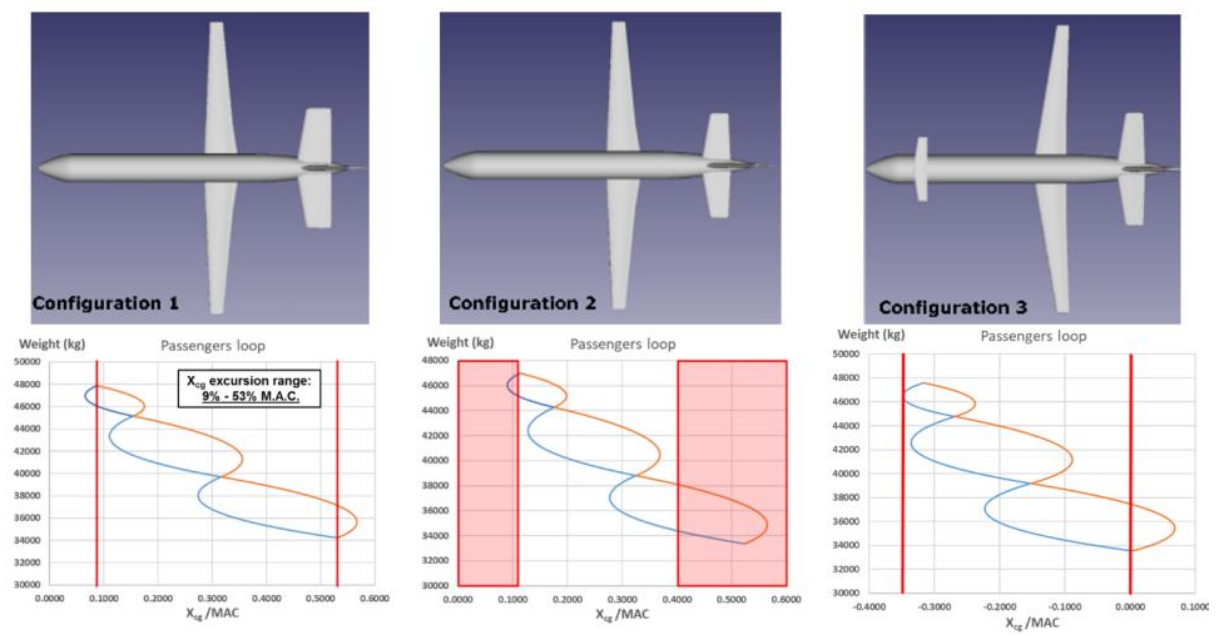

Fig. 2. Configuration comparison: upper view and boarding diagram to estimate the CG excursion starting from Operative Empty Weight CG position (the lowest weight condition) - blue line: front to rear pax. boarding; orange line: rear to front pax. boarding; red lines: imposed CG limitations.

To avoid a large horizontal tail area, a limitation on the center of gravity range has been imposed leading to the Configuration 2. This aircraft must operate within the imposed CG variation (11\% to $40 \% \mathrm{MAC})$. This allows to reach a higher cruise efficiency with respect to the first configuration $(\mathrm{E}=17.7)$, a lower maximum take-off weight and a slightly reduction of maximum lift coefficient at landing (see Table 1). Configuration 3 provides for a three lifting surfaces layout. In this configuration the $\mathrm{CG}$ range lies forward the mean aerodynamic chord leading edge (see Fig. 2). This latter allows to trim the aircraft with a reduced download on the horizontal tail, leading to a lower trim drag. This provides a higher cruise efficiency (18.4 with respect to 17.7 of the Configuration 2). The results achieved through the MDO process, have highlighted that the three-lifting surface is the most promising layout for such a high capacity turboprop platform.
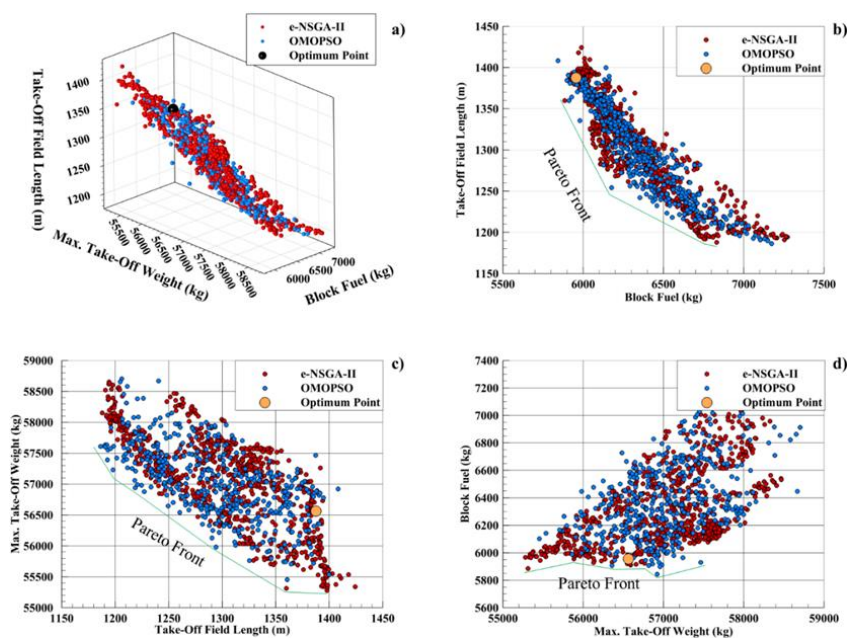

Fig. 3. Three Lifting Surface configuration: Pareto fronts and selected "optimum" point 
Table 1. Configurations comparison.

\begin{tabular}{|r|c|c|c|c|}
\hline & Loop 1 & Config. 1 & Config. 2 & Config. 3 \\
\hline $\mathbf{W}_{\text {To }}(\mathbf{k g})$ & 54410 & 54976 & 54105 & 54849 \\
\hline Cruise Efficiency & 16.7 & 17.3 & 17.7 & 18.4 \\
\hline $\mathbf{C}_{\text {Lmax }}$ clean & 1.53 & 1.70 & 1.70 & 1.91 \\
\hline CLmax $_{\text {TO }}$ & 2.15 & 2.63 & 2.63 & 2.90 \\
\hline $\mathbf{C}_{\text {Lmax }}$ LND & 2.30 & 3.19 & 3.09 & 3.40 \\
\hline $\mathbf{S}_{\mathbf{w}}\left(\mathbf{m}^{\mathbf{2}}\right)$ & 105 & 103 & 105 & 98.6 \\
\hline Max. Cruise Mach & 0.61 & 0.63 & 0.64 & 0.66 \\
\hline
\end{tabular}

\section{Aerodynamic assessment of the three-lifting surface aircraft}

Once the configuration has been defined, the DAF research group had in charge the aerodynamic assessment of the proposed concept, in particular of the longitudinal stability of the three-lifting surface aircraft for both high-lift and clean conditions.

The aerodynamic assessment of the aircraft longitudinal characteristics has highlighted some criticalities dealing with the interference effects of a three-lifting surface aircraft at full flap conditions [2]. Several CFD-RANS simulations have been performed on the wingbody and wing-body plus canard configuration to assess the effects of the canard wake at full flap condition $\left(\delta_{\mathrm{Fw}}=35^{\circ}\right.$ and $\left.\delta_{\mathrm{Fc}}=25^{\circ}\right)$. These analyses have highlighted that the canard, when its flaps are deployed, introduces a strong downwash on the main wing leading to a lift loss in the inner wing sections as shown by the wing lift distribution illustrated in Fig. 4 (left). On the other hand, the canard tip vortex introduces an upwash on the outer wing sections leading to a premature stall at the wing tip region, these effects are highlighted by the wing loading distribution shown in Fig. 4 (right). To mitigate this effect, a design study has been performed by varying the wing twist distribution in order to compensate both the lack and the excess of lift in the inner and outer wing region respectively. The wing twist has been changed accordingly to the estimation of the deficit in wing local angle of attack. This latter has been derived by comparing the wing lift distribution of the canard on and canard off configurations. The beneficial effects of this design study are highlighted in Fig. 4 (bottom), where the lift curves of the wing-body plus canard configuration with the initial (black line) and the optimized (red line) twist distributions are illustrated. Thanks to the wing twist optimization, it has been possible to increase the stall angle of about $5^{\circ}$ gaining a delta in $C_{\text {Lmax }}$ of about 0.4. At full flap condition, the canard wake has also a detrimental effect on the aircraft longitudinal stability. At low Angle of Attack (AoA), from $0^{\circ}$ up to $6^{\circ}$, the canard wake moves from the lower to the upper side of the horizontal tail, as highlighted in Fig. 5 (right). In this range of AoA the aircraft stability is reduced, as shown by the black solid line in the pitching moment breakdown chart of Fig. 5 (left). This interference effect was expected due to the reduced vertical stagger between the tail plane and the canard; however, it was supposed to be not so critical (considering the preliminary results carried out with low/medium fidelity tools like semi-empirical approaches, panel code approach and vortex lattice methods). This issue has been mitigated by shifting up the canard surface of about $0.3 \mathrm{~m}$ along the vertical axis, by adding a fairing to reduce the interference effects of the canard-fuselage junction, 
and by reducing the canard flap deflection from $25^{\circ}$ to $15^{\circ}$. These modifications have led to no longer unstable aircraft at full flap conditions, see [2]. Once those two main issues have been assessed, the complete aircraft trimmed drag polar curves have been derived thanks to high fidelity CFD-RANS analyses. This has been a joint work among DAF group and CIRA. CIRA has assessed the laminar flow on the main wing showing that the laminar flow allows to save up to 20 drag counts with respect to a fully turbulent wing.

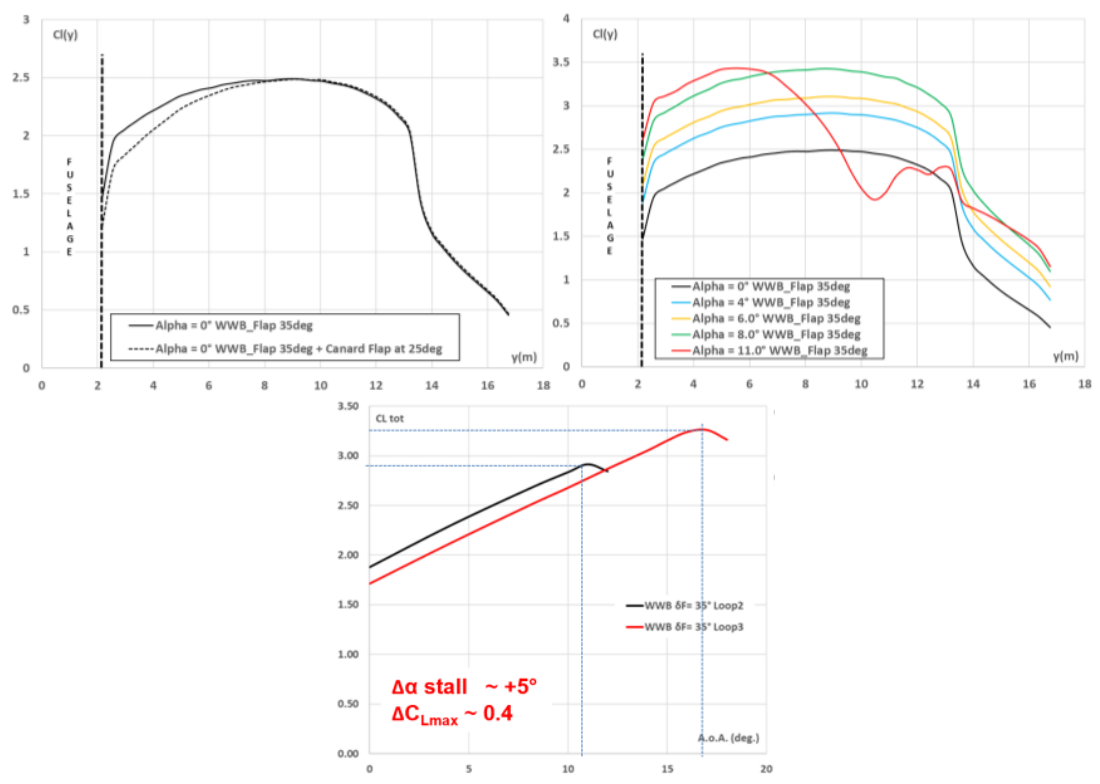

Fig. 4. Wing lift characteristics: left - comparison between span loading of canard on (dashed line) and canard off (solid line) at $\mathrm{AoA}=0^{\circ}$; right - wing lift distribution till the stall occurrence (red line); bottom - lift curve comparison before and after the wing twist optimization.
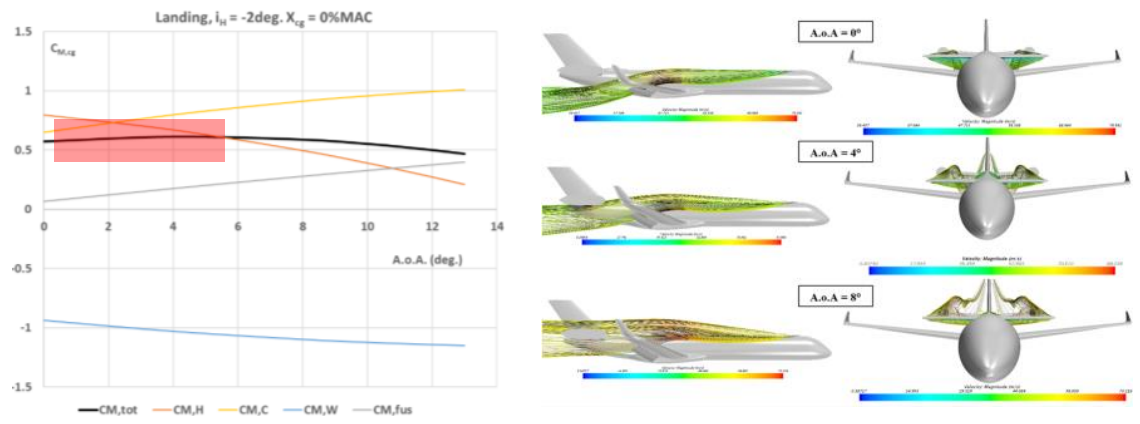

Fig. 5. Complete aircraft pitching moment curve breakdown at landing configuration (left); streamlines to highlight the canard wake impinging the horizontal tailplane at several AoA (right).

\section{Performance estimation: comparison with reference $\mathbf{R J}$}

Based on the drag polar curves and thanks to a simulation-based approach, the aircraft flight performance has been estimated. For the sake of brevity, in this section the main results will be shown in form of comparative table. The mission profile analysis has the key-role of investigating the behaviour of the aircraft during a specific mission. The main goals are to evaluate the fuel consumption during the whole mission and the time required 
to cover the assigned range. Results have been compared to a reference regional jet: the Airbus A220 (former Bombardier CS300). In this section the results on a typical mission of about $400 \mathrm{~nm}$ are shown, the analysis has been performed also on a longer mission range of about $1600 \mathrm{~nm}$.

Table 4. Typical mission (400nm @130pax): comparison between IRON and A220 (former CS300).

\begin{tabular}{|c|c|c|c|}
\hline & $\begin{array}{c}\text { Off } \\
\text { Design }\end{array}$ & $\begin{array}{l}\text { Laminar } \\
\text { Flow }\end{array}$ & $\begin{array}{c}\text { A220 } \\
\text { (CS300) }\end{array}$ \\
\hline Block Time (min) & $\begin{array}{c}74 \\
(+19.35 \%) \\
\end{array}$ & $\begin{array}{c}74 \\
(+19.35 \%) \\
\end{array}$ & 62 \\
\hline Block Fuel (kg) & $\begin{array}{c}1664 \\
(-19.61 \%)\end{array}$ & $\begin{array}{c}1606 \\
(-22.40 \%)\end{array}$ & 2070 \\
\hline Total DOC $\left(\mathbb{C} /\left(\mathrm{nm}^{*}\right.\right.$ seat $\left.)\right)$ & $\begin{array}{c}21.63 \\
(-6.28 \%)\end{array}$ & $\begin{array}{c}21.52 \\
(-6.75 \%)\end{array}$ & 23.08 \\
\hline Cash DOC $\left(\mathbb{C} /\left(\mathrm{nm}^{*}\right.\right.$ seat $\left.)\right)$ & $\begin{array}{c}12.44 \\
(-5.83 \%)\end{array}$ & $\begin{array}{c}12.36 \\
(-6.42 \%)\end{array}$ & 13.21 \\
\hline
\end{tabular}

The innovative turboprop platform, compared to the reference RJ, could bring to the possibility to save up from 22 up to $28 \%$ of the block fuel (according to the considered mission profile, 400 or $1600 \mathrm{~nm}$ respectively). About $20 \%$ of this fuel saving is reached thanks to the turboprop engine SFC. But, the remaining 2 up to $8 \%$ has been achieved by improving the aerodynamics of a high-capacity turboprop platform.

\section{Conclusion}

The feasibility study of a high-capacity turboprop has been faced demonstrating that it would be possible to design an innovative layout that could be competitive with respect to RJs on short/medium range. The adoption of an innovative turboprop platform could bring to the possibility to save up from 22 up to $28 \%$ of the block fuel burnt and to a reduction of 5 up to $6 \%$ of in total cash and total DOC with respect a RJ aircraft. About the $20 \%$ of fuel savings comes from the turboprop engine SFC while the remaining 2 up to $8 \%$ can be achieved by improving the aerodynamics of a high-capacity turboprop platform. The most promising configuration has been identified in a three-lifting surface one. However, the three-lifting surface aircraft introduces several criticalities dealing with the canard wake and its tip vortex effects, that must be well investigated in designing such a configuration.

The project leading to these results (IRON project) has received funding from the Clean Sky 2 Joint Undertaking under the European Union's Horizon H2020 research and innovation program under Grant Agreement $n^{\circ} 807089$ REG GAM 2018. The authors are grateful to the partners of the IRON consortium for their contributions and feedback.

\section{References}

[1] F. Nicolosi, S. Corcione, P. Della Vecchia, V. Trifari and M. Ruocco, Aerodynamic Design and Analysis of an Innovative Regional Turboprop Configuration, Proceedings of the 31st ICAS Conference (International Council of the Aeronautical Sciences), Belo Horizonte, Brazil, 2018. ISBN: 978-3-932182-88-4

[2] S. Corcione, F. Nicolosi, P. Della Vecchia, D. Ciliberti and V. Cusati, High Lift Aerodynamic characteristics of a Three Lifting Surfaces Turboprop Aircraft, AIAA Aviation 2019 Forum,1721 June 2019, Dallas, Texas, https://doi.org/10.2514/6.2019-2884 